\title{
Self-admission to inpatient treatment for patients with anorexia nervosa: The patient's perspective
}

\author{
Mattias Strand, MD 1,2 | Cynthia M. Bulik, $\mathrm{PhD}^{3,4,5}$ | \\ Yvonne von Hausswolff-Juhlin, MD, PhD ${ }^{1,2}$ ～Sanna A. Gustafsson, $\mathrm{PhD}^{6}$
}

${ }^{1}$ Stockholm Centre for Eating Disorders, Stockholm, Sweden

${ }^{2}$ Centre for Psychiatry Research, Department of Clinical Neuroscience, Karolinska Institutet, Stockholm, Sweden

${ }^{3}$ Department of Medical Epidemiology and Biostatistics, Karolinska Institutet,

Stockholm, Sweden

${ }^{4}$ Department of Psychiatry, University of North Carolina at Chapel Hill, Chapel Hill, North Carolina

${ }^{5}$ Department of Nutrition, University of North Carolina at Chapel Hill, Chapel Hill, North Carolina

${ }^{6}$ Faculty of Medicine and Health, Psychiatric Research Centre, Örebro University, Örebro, Sweden

Correspondence

M. Strand; Stockholm Centre for Eating Disorders, Stockholm, Sweden

Email: mattias.strand@ki.se

\begin{abstract}
The aim of the present study was to explore patients' experiences of participating in a selfadmission program at a specialist eating disorders clinic. Sixteen adult program participants with a diagnosis of anorexia nervosa were interviewed at 6 months about their experiences in the selfadmission program. A qualitative content analysis approach was applied to identify recurring themes. Four themes were identified: Agency and Flexibility, Functions, Barriers, and Applicability. Participants used self-admission to boost healthy behaviors, to prevent deterioration, to forestall the need for longer periods of hospitalizations, and to get a break from overwhelming demands. Quick access to brief admissions provides a safety net that can increase feelings of security in everyday life, even for patients who do not actually make use of the opportunity to self-admit. It also provided relief to participants' relatives. Furthermore, participants experienced that selfadmission may foster agency and motivation. However, the model also requires a certain level of maturity and an encouraging environment to overcome barriers that could otherwise hinder optimal use, such as ambivalence in asking for help. Informants experienced that self-admission could allow them to gain greater insight into their disease process, take greater responsibility for their recovery, and transform their health care from crisis-driven to proactive. By offering a shift in perspective on help-seeking and participation, self-admission may potentially strengthen participants' internal responsibility for their treatment and promote partnership in treatment.
\end{abstract}

\section{KEYWORDS}

anorexia nervosa, eating disorders, inpatients, patient admissions, patient-centered care, patient participation, voluntary admissions

\section{I INTRODUCTION}

The majority of patients with anorexia nervosa (AN) can be successfully treated in an outpatient setting (Keel \& Brown, 2010; van Son, van Hoeken, van Furth, Donker, \& Hoek, 2010). However, the course of AN can also be prolonged and may lead to enduring disability (Wentz, Gillberg, Anckarsäter, Gillberg, \& Råstam, 2009). Some patients require lengthy periods of inpatient treatment and relapse after discharge is common (Eckert, Halmi, Marchi, Grove, \& Crosby, 1995). Unfortunately, there is still little evidence concerning the optimal model of inpatient care for patients suffering from AN (Hay \& Touyz, 2012) and unlike psychiatry in general, the hospitalization rate for this group has not been markedly reduced in the past decades (Papadopoulos, Ekbom, Brandt, \& Ekselius, 2009).
Starting in 2014, Stockholm Centre for Eating Disorders (SCÄ) in Stockholm, Sweden began offering self-admission to patients with an eating disorder (ED). The details of this model have been described in the "An Idea Worth Researching" section of the International Journal of Eating Disorders (Strand, Gustafsson, Bulik, \& von Hausswolff-Juhlin, 2015) and are recapitulated in the Section 2 below. In self-admission, patients who are well-known to a service who have high previous utilization of health care are offered the possibility to self-admit to the inpatient ward for up to 7 days without having their motive for admission questioned. Patients are free to admit themselves because of deteriorating mental health, acute stress, or any other reason. The patients decide when they want to admit themselves and can selfdischarge at any time. The purpose behind the self-admission model is to increase the availability of specialist inpatient care, to avoid stressful 
and possibly destructive visits to the emergency service, and to decrease total inpatient care utilization. Patients offered a contract for self-admission usually have a history of repeated and prolonged hospitalizations. By encouraging them to monitor their own mental health status and allowing them to seek help swiftly when they are feeling poorly, the delay from first signs of deterioration to admission can be minimized and full-blown relapse may be avoided, ultimately reducing the total time spent in hospital. Although participants will most likely present with a clinical picture of treatment-refractory $A N$, the program does not exclusively target this group; patients with long-standing bulimia nervosa, purging disorder, or other presentations may be able to make use of the concept as well.

In Norway, self-admission to inpatient treatment for psychiatric patients has been in place for approximately 10 years, with promising results (Strand \& von Hausswolff-Juhlin, 2015). Self-admission has led to increased patient participation and compliance, strengthened the patients' abilities to handle their symptoms and their everyday life, and clearly reduced the total time spent in inpatient care. The total time spent in involuntary inpatient care was also reduced. Participants highlighted how self-admission served as a safety-valve, transforming the inpatient ward into a safe haven for them (Olsø et al., 2016). Patients with a contract for self-admission displayed greater confidence in using coping strategies to deal with their illness and a broader repertoire of cognitive tools (Rise et al., 2014). Furthermore, they expressed a stronger tendency of using these skills "in order to grow, to break barriers and to take opportunities" (Rise et al., 2014, p. 5).

The Norwegian programs of self-admission have primarily targeted patients with long-standing psychotic disorders, such as schizophrenia, or bipolar disorder. However, patients with long-standing AN are comparable to patients with schizophrenia or severe depression in terms of illness, quality of life, and health care utilization (Arkell \& Robinson, 2008) and could thus also be expected to benefit from self-admission. The brief nature of these admissions does not allow for full-scale weight restoration or achievement of other long-term treatment goals. Instead, self-admission is probably best used as booster opportunity or for providing a short respite from stressful life situations where the risk of relapse is elevated. In this way, offering inpatient treatment as a preemptory tool to be made use of rather than as a "necessary evil" when severe mental breakdown has already occurred can hopefully increase patient participation, agency, self-awareness, and autonomy.

The primary objective of this study was to explore patients' experiences of participating in a self-admission program at a specialist ED clinic.

\section{2 | METHODS}

\subsection{Setting and participants}

This study took place at SCÄ, which is a public specialist ED clinic run by the Stockholm County Council. The catchment area is Metropolitan Stockholm in Sweden with a population of 2.2 million. At the adult inpatient ward, two beds out of eleven are reserved for patients with a contract for self-admission. All regular admissions to the remaining nine beds are initiated by the SCÄ outpatient units on a strictly elective basis. Since regular admissions to specialist inpatient ED treatment are often prolonged, the patient turnover at the ward is low and there is typically a wait of several weeks for these elective admissions. No emergency admissions are available at SCÄ; instead, emergency cases are routinely handled by general psychiatry or somatic health care. Treatment at SCÄ is publicly funded, with only minor patient fees in consonance with all Swedish public health care; the per diem patient fee for inpatient treatment (regular as well as self-admission) is currently equivalent to 10 USD.

To be eligible for the self-admission program, patients must maintain continuous treatment contact at the adult outpatient or day treatment units. They must have had at least one treatment episode in the adult inpatient ward during the past 3 years and thus be familiar with the inpatient treatment framework and routines. Exclusion criteria in the self-admission program are current suicidal or self-injurious behavior and/or the presence of untreated substance use. Participants can admit themselves at will for a maximum of 7 days by contacting the ward directly. If both designated beds are already occupied, a waiting list is established. Participants are also free to discharge whenever they want. The model is designed to allow for maximum flexibility, so that participants can for example go to work or school during the day and only eat certain meals at the ward, etc. The self-admission contract is valid for one year, with the possibility of renewal annually. Selfadmission is constructed as an add-on treatment option and regular admission is still available for the participants if necessary.

The informants in this study were 15 women and one man with a mean age of 31 years (range 18-56, median 27) enrolled in the selfadmission program at SCÄ. All 18 patients with a self-admission contract were asked to be interviewed about their participation after 6 months in the program and all but two agreed to participate in the interview study. Written consent was obtained. Although this was not an explicit requirement for inclusion in the program, all informants were diagnosed with $\mathrm{AN}$, in some cases in partial remission as defined in the Diagnostic and Statistical Manual of Mental Disorders, Fifth Edition since they had normalized their weight during treatment. They had been suffering from an ED for a mean of 15 years (range 3-42, median 11) at inclusion. The informants' mean body mass index (BMI) at inclusion was $15.8 \mathrm{~kg} / \mathrm{m}^{2}$ (range 10.2-20.6, median 15.5). Thirteen informants were offered their contract upon discharge after a regular inpatient episode, whereas three of them were included during outpatient or day treatment. During their first 6 months in the program, 14 informants had made use of the opportunity to self-admit, while two of them had not. Of those who had self-admitted, five informants had done so only once whereas two of them had made use of the opportunity 14 times (mean 5.2, median 3).

\subsection{Procedure}

All informants were interviewed at 6 months after receiving their contracts for self-admission, regardless of whether they had actually selfadmitted. A semi-structured interview manual was prepared, defining the research questions that were to be investigated during the 
interviews. Certain questions in the interview manual differed based on the individual informants' use of their contract; for example, those informants who had never actually self-admitted during their time in the program where asked specific questions about the reasons behind this, whereas they could naturally not be interviewed about their experiences of the admission process, etc. All interviews were conducted face-to-face by a single interviewer (MS), who does clinical work at SCÄ, but is not directly involved in the treatment at the inpatient ward. During the interviews, an open interview technique was applied whereby all informants were asked the same opening question ("Could you please tell me about your experiences so far in the self-admission program?"), after which the informants were asked open-ended followup questions appropriate to the topics brought up in the conversation ("Are there any positive aspects of self-admission?", "Are there any negative aspects of self-admission?", "Has your participation in the program affected your everyday life in any way?", etc.). All interviews were tape recorded and transcribed verbatim by the interviewer. During transcription, all statements involving personal details that could possibly reveal the identity of the informants to those who know them well (e.g., the staff at the inpatient ward) were modified and anonymized; this procedure was explained to all informants before the interviews in order for them to be able to speak as freely as possible about their experiences in the program.

All procedures were conducted in accordance with the Helsinki declaration. The study was approved by the Regional Ethical Review Board in Stockholm, Sweden. The study protocol is registered at ClinicITrials.gov as ID: NCT02937259.

\section{3 | Data analysis}

The verbatim-transcribed interviews were analyzed using the qualitative analysis software program NVivo 11. A qualitative content analysis approach was applied (Hsieh \& Shannon, 2005) whereby those excerpts of the interview text that was regarded as meaningful in relation to the research questions were coded and labelled according to a "bottom-up" principle. In an initial round of content analysis, the interviews were coded freely by the first author (MS), yielding 72 separate coding categories. The two authors mainly responsible for data coding (MS and SAG) then jointly reviewed these categories and grouped them into main themes and subthemes. There were no predefined criteria (e.g., number of informant statements needed) to aid in determining what would constitute a separate theme or subtheme; instead, after the initial "bottom-up" approach had yielded a number of statement categories, meaningful clusters were identified and developed inductively by analyzing patterns and interrelations and labelled so as to reflect nuances within the themes. Using this preliminary data coding scheme, MS and SAG separately reanalyzed the interviews and modified the coding scheme accordingly, adding newfound themes and regrouping the theme hierarchy when needed. MS and SAG eventually agreed on a data coding scheme with four main themes, each with a number of subthemes (see "Section 3"). With this updated data coding scheme, MS and SAG went back and separately re-coded the interviews in a "top-down" approach to make sure that the coding scheme was now accurate and reliable. At this point, an inter-rater reliability of 91\% was reached. For those statements were MS and SAG differed in their coding, consensus was reached through discussion. After interview 12, no new codes emerged and the interview material was thus considered as saturated; i.e., even if more interview material would increase the amount of information, it was unlikely to lead to the emergence of new topical themes.

The quotes used in the Results section were translated from Swedish into English by the first author (MS) and verified by the rest of the author group.

\section{3 | RESULTS}

Four themes and 16 subthemes emerged from the interview data (see Figure 1). Informant quotes illustrating the themes described below are presented in Tables 1-4. Overall, the informants were satisfied with their participation in the self-admission program and all of them would recommend enrollment to other patients in similar situations. Informants valued the agency and flexibility inherent to the model and described how self-admission could serve various functions depending on the individual's life situation. They also described contextual requirements that had to be met in order to benefit from the model in a constructive way, as well as some important barriers to overcome.

\section{1 | Agency and flexibility}

A number of recurring statements were sorted under a theme describing the advantages and perils of the increased flexibility, responsibility and agency inherent to the self-admission model. Two subthemes were identified (see Figure 1 and Table 1).

"Agency" was raised as a central feature of the model. Some informants felt that this cooperative aspect opened up entirely new possibilities for them in their treatment (quotes 1.1 and 1.2). However, some informants also mentioned risks associated with having too much decision-making power (quotes 1.3 and 1.4).

"Flexibility" was also seen as a central aspect of the model, allowing participants to tailor the inpatient treatment according to their current life situation and to their most urgent needs (quotes 1.5-1.7).

\subsection{Function of the self-admission program}

Six subthemes related to the function of self-admission emerged (see Figure 1 and Table 2). "Security" was raised both by informants who had made use of their self-admission contracts and by those who had never actually availed themselves of the opportunity to self-admit; even the latter group saw this as an important function of the model that allowed them to feel safer and more secure in their everyday lives by providing an "alternative" or a "safety net" (quotes 2.1 and 2.2).

"Boosting healthy routines" was raised as a straightforward way of making use of the brevity of the self-admission episodes (quotes 2.3 and 2.4). "Preventing deterioration and prolonged hospital admissions" was another recurring subtheme. Here, informants noted that while they were not eager to spend time at the ward, it was worth being 


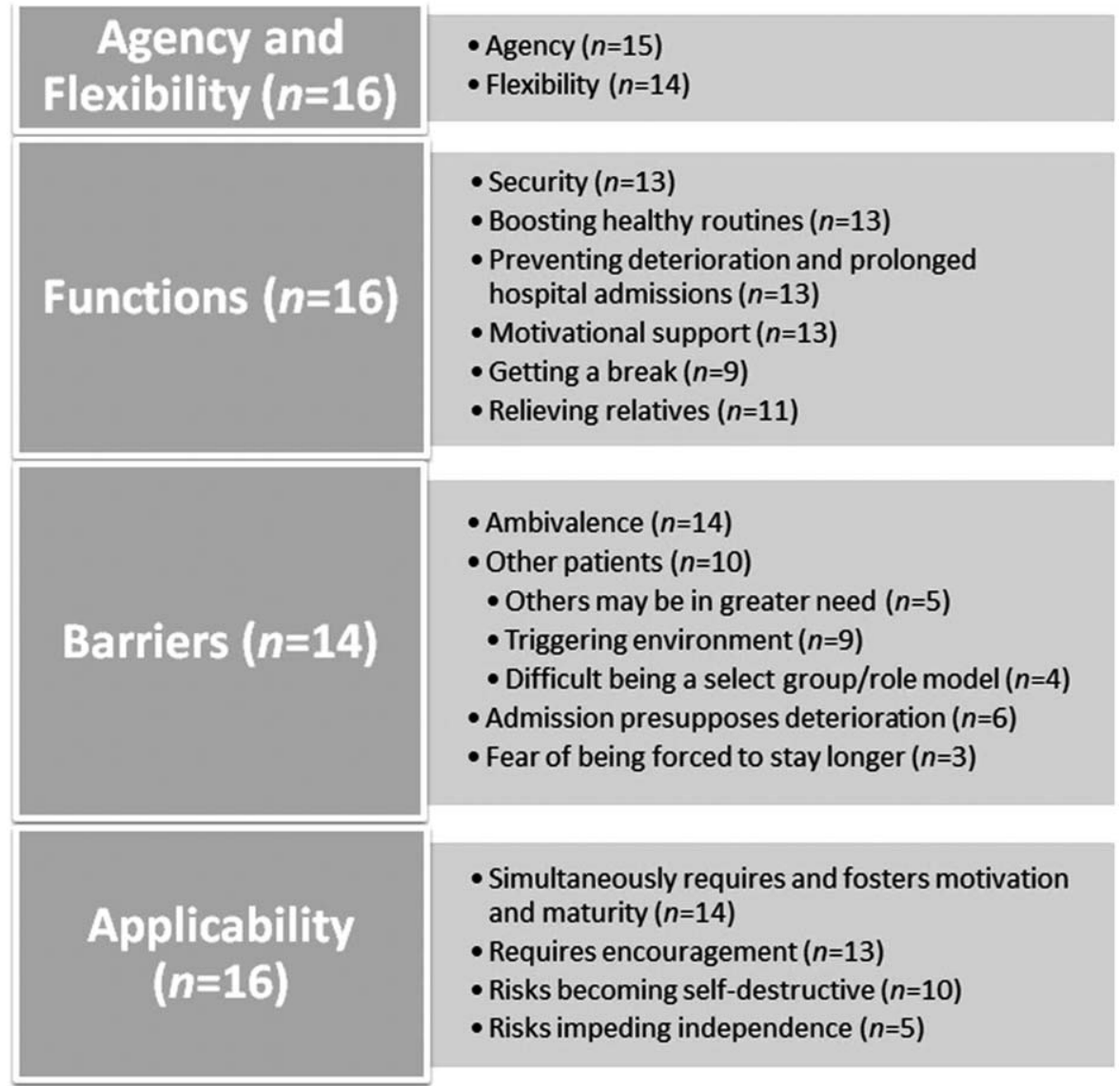

FIGURE 1 Themes and subthemes

there for a brief period of time if they could thereby avoid longer periods of hospitalization (quotes 2.5 and 2.6).

"Motivational support" involved descriptions of how the thought of admission could help informants to stay focused and keep making behavioral changes at home (quotes 2.7 and 2.8). In addition, a few informants mentioned using self-admission very specifically as a way of motivating themselves in expanding their horizon and doing things that had previously been difficult; e.g., daring to go on holiday trips abroad because they knew that if it did not go well, they would have quick access to inpatient treatment when they got back home.

"Getting a break" was discussed in a broader sense (quote 2.9) as well as in very specific ways (quote 2.10). For some, the ward could provide respite from overwhelming everyday demands (quote 2.11).

Most informants had explained the model to their close relatives and accordingly "Relieving relatives" was a recurring function cited in the interviews (quotes 2.12 and 2.13). Knowing that there was quick access to inpatient treatment could increase feelings of safety in loved ones, who may have been actively involved in securing accurate treatment for the informants over many years.

\section{3 | Barriers}

Four subthemes related to barriers in using self-admission emerged (see Figure 1 and Table 3). "Ambivalence" was a recurring subtheme, usually mentioned as being an integral part of AN (quotes 3.1-3.3). "Other patients" was subdivided into three further subthemes (see Figure 1). Several informants mentioned thinking about whether their coparticipants may need the hospital beds more than themselves (quote 3.4). Furthermore, several informants mentioned that the ward climate is not always supportive and that other patients can have a triggering effect (quote 3.5). One informant also mentioned that returning to the ward could have a disheartening effect on a larger scale (quote 3.6). Some informants brought up negative feelings of being a brief "visitor" at the ward and "disturbing" the regular patients. The fact that informants had actually chosen for themselves to be admitted could lead to contradictory feelings (quote 3.7).

"Admission presupposes deterioration" involved different nuances. Several informants said that they feel that it has to be "understandable" to others (staff members, other patients, etc.) that they need help when they do admit themselves, i.e., there needs to be visible signs of deterioration. They also mentioned experiencing AN as having a will of its' own, "wanting" to squeeze the last drops out of them before they could seek help (quote 3.8). Others brought up feelings of failure on returning to the ward (quote 3.9). Some informants described how others sometimes automatically assumed that they admitted themselves because of deteriorating health, which was not necessarily the case (quote 3.10). 
TABLE 1 Informant quotes on agency and flexibility

\section{Agency}

1.1 Patient 6 (had not self-admitted): It's very important for me. I have a high level of integrity. And that's what's so fantastic about this: it's a whole new approach.

1.2 Patient 2: Now when you talk to a doctor at the ward, they listen to what you say and actually reflect upon it instead of just looking at you like you're trying to fool them or like you have ulterior motives.

1.3 Patient 1: If you could decide everything for yourself when you're at the ward it would be just like being at home, and then what's the point?

1.4 Patient 15 (had not self-admitted): I get to decide a lot, but when it comes to my eating disorder I don't think I should have too much say, because it'll be bad for me.

\section{Flexibility}

1.5 Patient 13: I tested combining self-admission with my job training. I was at the ward, then I went to my workplace, then I came back to the ward.

1.6 Patient 5: It was also more of a freedom, not feeling that I had to sit there and watch the clock and be like 'right, three hours left until next meal' and then 'now it's two hours left'-that institutional feeling. This way, it's almost like going to work: you do what you're supposed to do and then you go home.

1.7 Patient 16: It felt good, because I could leave for a few hours every day. For my afternoon snack. So it wasn't as confined as I had thought. Those hours made a big difference, it wasn't like being at a closed ward.

"Fear of being forced to stay longer", finally, was brought up by a few informants who had previous experiences of compulsory inpatient treatment (quote 3.11).

\section{4 | Applicability}

A number of recurring statements were sorted under a broader theme describing for whom the informants believed self-admission was or was not applicable. Four subthemes emerged (see Figure 1 and Table 4).

Most of the informants discussed how the model relates to factors such as personal motivation, awareness, and maturity. Both the idea that a certain level of motivation and maturity is required in order to make constructive use of self-admission and the idea that the model could in and by itself foster such skills and traits were brought up, not seldom within the same statement. They were therefore merged into the single subtheme "Simultaneously requires and fosters motivation and maturity". A lack of motivation was seen as potentially dangerous in this context (quotes 4.1 and 4.2). However, several informants also discussed how the freedom intrinsic to self-admission could help to "bypass" aspects that had previously been problematic (quotes 4.3 and 4.4). Self-admission could help informants to practice self-awareness and active help-seeking (quotes 4.5 and 4.6). Several informants discussed how they felt that self-admission had promoted an ongoing process of shifting focus about what constitutes a life worth living and what goals are actually attainable (quotes 4.7 and 4.8).

"Requires encouragement" involved statements about how it was helpful to have somebody-usually a therapist but at times also a rela- tive or a fellow patient-who could remind them of the opportunity and encourage utilization, in order to get past the ambivalence and make use of self-admission as a resource (quotes 4.9 and 4.10).

Under "Risks becoming self-destructive", informants acknowledged that there could be a delicate balance between using the flexibility in a

TABLE 2 Informant quotes on the functions of self-admission Security

2.1 Patient 6 (had not self-admitted): I think this contract gives me an enormous sense of security because [.. . I can try on my own and if it doesn't work out, I always have a backup plan. You know you won't just fall headlong.

2.2 Patient 11: Yes, it affects my everyday life 24/7, just knowing that I have the opportunity.

\section{Boosting healthy routines}

2.3 Patient 5: Then I found myself in a situation where I didn't eat food, only liquid supplements. So l admitted myself for a week just to get out of that supplement swamp and start eating regular meals again. [...] That was really a good admission, probably my first sound admission where I've felt like I was actually 'on board' myself. I've been treated against my will a lot, but this time I really set a goal, totally focused on it and used this week to get back to regular meals again.

2.4 Patient 11: I've been to the ward just to update my sense of a regular portion size. And it works.

Preventing deterioration and prolonged hospital admissions

2.5 Patient 9: Before, whenever things started going downhill and it became more difficult to eat, it would get really bad before I could get help and then it's so much harder getting back up in the sadle again. But now it hasn't been that hard because l've been able to get back to my usual routines and stop these behaviors before they go too far.

2.6 Patient 11: I don't need to be there for eleven months-if I just ask for it in time, it can be eleven days instead. [. . .] If you just sacrifice two weeks, you gain ten months of freedom. It's a pretty big thing.

\section{Motivational support}

2.7 Patient 5: A few times this spring l've had minor setbacks. And so I've said to myself: - Do you want to go back to the ward again? Is that what you want?-No, no, no, I don't!-Well, then behave! [...] But at the same time, I know that if it doesn't work out, I can always go in for a 'quickie'.

2.8 Patient 11: The ward can be sort of a walking companion; I don't have to drag everything along all by myself. I'll do it on my own but I'm never alone, because the ward is there just in case.

\section{Getting a break}

2.9 Patient 14: To get just a few days at the ward - shutting the rest of the world out, handing over choices and letting go of control [...]. To be treated so fantastically was really valuable to me.

2.10 Patient 6 (had not self-admitted): Days when I just don't feel like cooking, I can go there and eat.

2.11 Patient 7: It was sort of an escape from my reality, a break from all those hard things, even if it's really difficult being at the ward. Just to get away from family demands and things like that.

Relieving relatives

2.12 Patient 1: [My mother] thinks it a relief knowing that I have this opportunity and that I don't have to be on an admission queve for several months if I actually need help right away.

2.13 Patient 3: My brother thinks it's one step towards a healthier future just being able to acknowledge that I need help. That's how he sees it. 
TABLE 3 Informant quotes on barriers

\section{Ambivalence}

3.1 Patient 2: God, l'd just like to admit myself and get some rest and get taken care of, but I discharge simply because I can. And now when I'm back-well, as you can see I'm in a wheel chair and I'm not allowed to do anything, so now I can only regret. . . Damn, why can't I have the strength to use self-admission in a sensible way?

3.2 Patient 5: There's always resistance to being admitted. But somehow you have to reason with yourself and realize that this isn't going to last. You need help to understand you need help. So I think about: Is this the life I want, [.. .] to never be able to eat food? What kind of life is that? [.. .] So you list your 'pros' and 'cons', and when the 'pros' get strong enough you seek help.

3.3 Patient 7: Of course, [self-admission] has been there as an option and somehow felt like a relief or a comfort, sort of. But at the same time it feels impossible.

\section{Other patients}

3.4 Patient 13: There's also a danger in knowing if someone else is on the waiting list, because then you might feel like that person probably needs it more and I'll just wait a little longer. Otherwise I'll feel guilty about someone else deteriorating and becoming really ill. [...] So I'll wait and call back the next day to see if they admitted themselves. You're sort of holding back all the time.

3.5 Patient 4: It's kind of like a microcosm in there. You're supposed to focus on your own treatment, but [.. .] it's impossible to not be affected by the atmosphere. Sometimes it's like a reality show in there, for real.

3.6 Patient 9: That's one of the most difficult things about being there: that you're sitting there with the same people-myself includedthat were there a year ago. Nine out of ten patients are the same and no one has made any progress. It makes you feel a bit hopeless.

3.7 Patient 1: To come there as a role model for health, so to speak, and admit voluntary to a ward that's currently infected with triggering and stuff like that. It can feel a bit paradoxical. [... I I haven't felt like some kind of sponsor of recovery when I use self-admission, but it's so obvious for all the others that might be there involuntarily that 'she comes here and admits herself by her own free will, how can you do that?' When I might still feel as bad as they do, only I want to get better.

\section{Admission pressuposes deterioration}

3.8 Patient 1: Such difficult challenges await at the ward, so the illness wants to take what it can get from you before you go against it.

3.9 Patient 10: It's like coming back with your tail between your legs. You've met the staff during the months you've been hospitalized and have gotten to know them. They've followed your journey [...] and then you return in slightly worse shape and it feels difficult having to meet them.

3.10 Patient 8: It was a bit awkward when I got there, because everybody reacted like: 'My god, it's so great that you come in when things are feeling hard.' '. . . Everyone assumed I was there because things were going bad [...] and then I started to feel like I had to confirm that.

Fear of being forced to stay longer

3.11 Patient 4: I'll always be a little scared, since my very first experience was that I wasn't actually allowed to discharge when I wanted to. So I'll always have that fear, unfortunately.

healthy and constructive way and allowing the ED more leeway (quotes 4.11 and 4.12). Some informants had noticed this risk and decided for themselves that they would not make use of this particular aspect of the self-admission model (quotes 4.13 and 4.14).
Finally, "Risks impeding independence" involved statements about fearing that self-admission may actually be potentially counterproductive by not allowing participants to fully let go of the inpatient ward in order to be able to recover (quotes 4.15 and 4.16).

\section{4 | DISCUSSION}

The present qualitative study reveals that participants with $A N$ in a self-admission program for patients at a specialist ED clinic experienced increased agency and motivation. However, the model also requires a certain level of maturity and an encouraging environment to overcome barriers that could otherwise hinder optimal use. Quick access to brief admissions can provide a safety net that may increase feelings of security in everyday life, even for patients who do not actually make use of the opportunity to self-admit. Participants used self-admission to boost healthy behaviors, to prevent deterioration, to forestall the need for longer periods of hospitalizations, and to get a break from overwhelming demands. It also provided relief to participants' relatives.

Whereas previous qualitative studies (Olsø et al., 2016; Rise et al. 2014) of patients with a psychotic or bipolar disorder who had been provided with a contract for self-admission reported only positive effects of the model, such as a broader repertoire of coping strategies and diminished feelings of resignation, in the present study there were also obstacles associated with the model that could hinder a constructive use of self-admission in AN patients. A major obstacle was participants' ambivalence in asking for help. While ambivalence is certainly not unique to patients with $\mathrm{AN}$, it does constitute a central aspect of the clinical picture that can often impede help-seeking and effective treatment engagement. Unfortunately, there is little evidence that commonly used models for enhancing motivation are helpful (Waller, 2012). However, while self-admission primarily provides a logistic tool rather than any specific treatment content, this study indicates that it may also change how patients grapple with their treatment on a more profound level. Self-admission could help to bypass certain aspects of inpatient treatment that had previously been problematic and make them redundant; e.g., participants reported how feelings of increased agency helped them to not relapse into a "tug of war" with the staff. By inevitably highlighting the participants' own choice to engage in inpatient treatment, the model may potentially foster reflection on subjective motives and barriers in asking for help and promote partnership between patient and clinicians instead of distrust. Informants reported that being given the opportunity to self-admit increased their sense of internal responsibility for their treatment and for making constructive use of the self-admission tool; this was reflected in their discussion of for whom the model is suitable and what requirements should ideally be met by the program participants. For many participants, meeting these requirements became a goal in itself. In part, these findings mirror the results in a recent study (Smith et al., 2016) on general patient experiences of specialist inpatient treatment for EDs, where topics such as sense of control, peer and staff relationships, and processes of self-discovery were raised. 
TABLE 4 Informant quotes on applicability

Simultaneously requires and fosters maturity and motivation

4.1 Patient 11: It's almost a survival mechanism: we [patients with anorexia nervosa] withdraw if we can. And you have to get over that threshold before you're given the opportunity of self-admission. Otherwise it becomes self-destructive.

4.2 Patient 5: I couldn't have had this contract ten years ago. Or, I could have had it but I wouldn't have used it. No way. It's like I have matured and realized that I have to ask myself: What are my goals? What do I want? And to reach those goals I may need to accept getting help.

4.3 Patient 15 (had not self-admitted): Once you've been offered a contract, it means that you have a very severe eating disorder. [...] My eating disorder doesn't have to prove anything.

4.4 Patient 13: Even if I don't do everything with joy when I'm [at the ward], I know that I'm doing it because I've chosen it myself. And then maybe I can show others that have even greater difficulties that it's possible to do it by your own free will. That it's ok to like the food.

4.5 Patient 5: I don't have to get to a BMI of 9; the point is to [admit yourself] in time. [.. .] It can be tough and there's no way around it. It's all about rising above it and accepting that you're doing it for your own sake. And I did.

4.6 Patient 3: For me, it has also been useful to ask for help. [. . . And it gets easier and easier every time. If you've done it once you know how it works.

4.7 Patient 13: It's now easier to dare believe that I could live a life in balance. Now I don't have to be so busy with maintaining a façade of healthiness any more-instead, I can work with what's really relevant.

4.8 Patient 2: My goal is just to find some kind of eating habits that will still be very disordered, that's just how it is. But just being able to eat and maintain a weight that I feel is ok and that isn't life threatening. [...] I used to think that this, the illness, was just make believe. That oh well, someone else will fix this for me. But I've realized that I have to take responsibility for feeding myself. [. . .] My wish is to maintain a decent everyday life with the help of selfadmission.

\section{Requires encouragement}

4.9 Patient 13: [My therapists] can sometimes urge me to come in for a week, because they recognize when I'm losing it. Because I still have an illusion of being able to cope.

4.10 Patient 15: Since it's kind of hard for me to make decisions on my own, it a relief that I don't have to. I don't think I would've contacted the ward if my therapist hadn't said 'come on, give them a call!'.

Risks becoming self-destructive

4.11 Patient 1: Perhaps you need to be careful with it because when you get to decide; very often it's the eating disorder talking. Maybe there needs to be an open dialogue so that the staff is alert about what is the illness and what is really favorable in going forward with this contract. [...] It's so easy to believe that 'well, she's here voluntarily, she really wants to get better' but that's not always the case.

4.12 Patient 4: Like Fatima... You could see she wasn't following the rules; she went out to eat every day-but no. And also, I follow her on Instagram so I know she didn't go out and eat. [. . .] For the staff to be fooled like that, I just think that's super weird.

4.13 Patient 15 (had not self-admitted): I think I'll try to be here as much as possible. So that every day looks the same, because that's how I need to run my life. It's good to allow for flexibility, absolutely, but I think I'll be here the whole time.

(continues)
TABLE 4 (continued)

\section{Simultaneously requires and fosters maturity and motivation}

4.14 Patient 3: Even if someone would say 'Can't we have lunch today?'-a friend or somebody - then I'd say 'No, I'm going to be at the ward'. It's better to eat there, that way I know I'll get what I need.

Risks impeding independence

4.15 Patient 7: One of the main reasons why I don't want to go back to the ward is that I've just started getting by on my own. [...] I'm scared to death that I would somehow become dependent again, dependent on others.

4.16 Patient 8: If you feel that you want to break free-from the illness, that is-then this is kind of like a shackle. You still have one foot in the ward.

Overall, the present findings must be interpreted with caution. It should be clear that the qualitative study design is not aimed at evaluating outcomes or establishing causality but at investigating patient experiences with regard to aspects such as trustworthiness and transferability. Although the present self-admission program is not exclusively targeted to AN patients, all informants in our sample had an AN diagnosis. This may affect transferability of the findings to groups with other EDs. Furthermore, some of the topics brought up by the informants may relate more to experiences of $\mathrm{AN}$ and $\mathrm{AN}$ treatment in general than to self-admission specifically-e.g., problems associated with ambivalence and the ego-syntonicity of symptoms in AN are certainly not unique to the self-admission model. While previous studies have indicated that self-admission can be a helpful treatment tool in psychiatry (Strand \& von Hausswolff-Juhlin, 2015), it is unclear for whom the model is most suitable and what factors that impact this viability. In, for example, patients with psychotic or affective disorders-groups that have previously been targeted in general psychiatric self-admission programs-there may of course be within-group differences regarding cognitive deficits, executive functions, insight etc. that affect constructive use. This holds true for AN as well and needs to be explored further.

Nevertheless, the present results have several potential treatment implications. Self-admission may transform inpatient treatment from crisis-driven to proactive, promote help-seeking behavior, and help patients to gain greater insight into their disease process and take greater responsibility for their treatment. However, the ambivalence inherent to $\mathrm{AN}$ must be taken into consideration so that patients are provided with adequate support to use the model appropriately. Staff must also be aware of the difficult balance between promoting flexibility and enabling self-destructive choices. Furthermore, although this self-admission program does not exclusively target patients with a severe and enduring ED (SE-ED), several of the informants presented with a SE-ED picture. There is an urgent need of novel treatment approaches for patients with SE-ED and in this context self-admission may possibly aid in shifting from a recovery-focused approach to a case management approach with greater emphasis on patient participation, agency, and quality of life.

This study has a number of strengths and limitations. It is the first study to provide patients' experiences of participating in a self- 
admission program as part of a specialist ED treatment setting. As such, it also adds new knowledge on patient experiences of inpatient treatment for AN in general-a field where only a handful of studies exist (Smith et al., 2016). Participants were interviewed during ongoing program participation, thus allowing them to discuss their experiences in "real-time". The coding scheme used in data analysis was established through a joint procedure and a high inter-rater reliability was achieved. However, the main interviewer was employed at SCÄ (although in a different department) which could have affected participants' responses. Furthermore, while self-admission was offered as a part of publicly funded health care, standard patient fees for inpatient treatment were charged. None of the informants brought this up as an obstacle but it may nevertheless have affected their ability to make use of self-admission. In addition, most published studies on self-admission in psychiatry emanate from the Scandinavian countries or the Netherlands, which have a strong emphasis on public health care. Adaptations will be required for different health care systems. Even so, it may be hypothesized that by establishing a "fast lane" for high consumers of inpatient care and thereby removing them from the general admission queue, waiting lists may ultimately be reduced; this could prove to be an attractive model regardless of particular health care financing systems.

Whereas this study highlights self-admission as a viable and acceptable tool from the patients' perspective, data do not yet exist to determine whether the model affects health care utilization patterns or outcomes. Health economics analyses are necessary to ensure that the model is scalable.

In conclusion, this study is the first to provide patients' experiences of participating in a self-admission program as part of a specialist ED treatment setting. All participants were satisfied with the model and they would all recommend the program to fellow patients, although barriers were also identified. Self-admission can allow patients to gain greater insight into their disease process, take greater responsibility for their recovery, and transform their health care from crisis-driven to proactive. By offering a shift in perspective on help-seeking and participation, self-admission may strengthen participants' sense of internal responsibility for their treatment and promote partnership in treatment.

\section{REFERENCES}

Arkell, J., \& Robinson, P. (2008). A pilot case series using qualitative and quantitative methods: Biological, psychological and social outcome in severe and enduring eating disorder (anorexia nervosa). International Journal of Eating Disorder, 41, 650-656.
Eckert, E. D., Halmi, K. A., Marchi, P., Grove, W., \& Crosby, R. (1995). Ten-year follow-up of anorexia nervosa: Clinical course and outcome. Psychological Medicine, 25, 143-156. 1

Hay, P. J., Touyz, S., \& Sud, R. (2012). Treatment for severe and enduring anorexia nervosa: A review. Australian \& New Zealand Journal of Psychiatry, 46, 1136-1144. 12

Hsieh, H. F., \& Shannon, S. E. (2005). Three approaches to qualitative content analysis. Quality Health Research, 15, 1277-1288.

Keel, P. K., \& Brown, T. A. (2010). Update on course and outcome in eating disorders. International Journal of Eating Disorder, 43, 195-204.

Olsø, T. M., Gudde, C. B., Moljord, I. E., Evensen, G. H., Antonsen, D. Ø., \& Eriksen, L. (2016). More than just a bed: mental health service users' experiences of self-referral admission. International Journal of Mental Health System, 10, 11.

Papadopoulos, F. C., Ekbom, A., Brandt, L., \& Ekselius, L. (2009). Excess mortality, causes of death and prognostic factors in anorexia nervosa. British Journal of Psychiatry, 194, 10-17. 1

Rise, M. B., Evensen, G. H., Moljord, I. E., Rø, M., Bjørgen, D., \& Eriksen, L. (2014). How do patients with severe mental diagnosis cope in everyday life-A qualitative study comparing patients' experiences of self-referral inpatient treatment with treatment as usual? BMC Health Survey \& Research, 14, 347.

Smith, V., Chouliara, Z., Morris, P. G., Collin, P., Power, K., Yellowlees, A., Grierson, D., et al. (2016). The experience of specialist inpatient treatment for anorexia nervosa: A qualitative study from adult patients' perspective. Journal of Health Psychology, 21, 16-27.

Strand, M., Gustafsson, S. A., Bulik, C. M., \& von Hausswolff-Juhlin, Y. (2015). Patient-controlled hospital admission: A novel concept in the treatment of severe eating disorders. International Journal of Eating Disorder, 48, 842-844.

Strand, M., \& von Hausswolff-Juhlin, Y. (2015). Patient-controlled hospital admission in psychiatry: A systematic review. Norwegian Journal of Psychiatry, 69, 674-686. 8

van Son, G. E., van Hoeken, D., van Furth, E. F., Donker, G. A., \& Hoek, H. W. (2010). Course and outcome of eating disorders in a primary carebased cohort. International Journal of Eating Disorder, 43, 130-138.

Waller, G. (2012). The myths of motivation: Time for a fresh look at some received wisdom in the eating disorders? International Journal of Eating Disorder, 45, 1-16.

Wentz, E., Gillberg, I. C., Anckarsäter, H., Gillberg, C., \& Råstam, M. (2009). Adolescent-onset anorexia nervosa: 18-year outcome. British Journal of Psychiatry, 194, 168-174. 2

How to cite this article: Strand M, Bulik CM, von HausswolffJuhlin Y, and Gustafsson SA. Self-admission to inpatient treatment for patients with anorexia nervosa: the patient's perspective. Int J Eat Disord. 2017;50:398-405. https://doi.org/ 10.1002/eat.22659 